\title{
Adaptive QoS and Handover Issues in Wireless Multimedia Networks Using a Dynamic Adaptive Architecture: DYNAA
}

\author{
Rola Naja and Samir Tohmé \\ Computer and Network Departement \\ Ecole Nationale Supérieure des Télecommunications \\ 46, Barrault Street, 75634 Paris \\ \{rola.naja,samir.tohme\}@enst.fr
}

\begin{abstract}
Adaptive Multimedia services are promising in wireless mobile networks since they can improve the quality of service (QoS). In our paper, we propose a dynamic adaptive architecture DYNAA which is based on a call admission control (CAC) and bandwidth adaptation algorithms. The architecture proposed manages to adapt dynamically the bandwidth adaptation to the user's mobility and the traffic load. DYNAA tries to establish an application-network collaboration that can cope with high variability in network conditions but can continue to transport multimedia content. Simulation results show the performance of the proposed scheme.
\end{abstract}

Keywords: Call admission control, adaptive quality of service, handover.

\section{INTRODUCTION}

In an end-to end QoS framework for multimedia wireless mobile systems, the major issue to be addressed is the high level of fluctuation in resource availability due mainly to mobility. There is a growing consensus that adaptive quality of service presents a viable approach to this issue. Hence, QoS provisioning is the responsibility of the network and the application in order to deliver multimedia content to a wireless mobile terminal in the most acceptable form. For adaptive multimedia services, the existing QoS parameters become very trivial to be guaranteed. The reason is

The work reported in this paper has been supported by the ITEA AMBIENCE project.

The original version of this chapter was revised: The copyright line was incorrect. This has been corrected. The Erratum to this chapter is available at DOI: 10.1007/978-0-387-35618-1_37 
that the adaptive framework moves into the problem of the bandwidth degradation caused by adaptation. Note that degradation is obtained when the assigned bandwidth is less than the required bandwidth.

A new QoS parameter, the Degradation Period Ratio $D P R$, is proposed in [2]. It represents the portion of a call's lifetime that a call is degraded. However, DPR does not characterize the bandwidth degradation. In order to fully characterize the bandwidth degradation and to provide better QoS to service users, the authors in $[3,6]$ propose two novel QoS parameters: the degradation ratio $D R$ and the degradation degree $D D$, which designate respectively the frequency and the degree of degradation.

In the classic adaptive framework, the call dropping probability $P_{d r o p}$ becomes very trivial to be guaranteed at the expense of the application degradation. It is true that the forced termination of a call is a very frustrating phenomenon that may happen to a user. However, service degradation can be very annoying especially when that arrives frequently. One of the critical tasks of a mobile computing environment is to prevent frequent adaptation, by reducing $D D$ and $D R$, due to the dynamics of resource and mobility of flows while still optimizing the network performance (i.e. still having $P_{\text {drop }}$ less than the required $P_{\text {drop }}\left(P_{\text {drop,qos }}\right)$ ). Another important task is to take into account the current load conditions when adapting the calls' bandwidth. Even, if the HO load decreases, CAC in [6] always accepts the HO request and that leads to the degradation of other calls in order to satisfy the incoming request. The above mentioned tasks are achieved in our proposed scheme DYNAA. In order to reflect the current load network conditions, DYNAA dynamically adapts the amount of bandwidth's adaptation based on the current network conditions (based on the average $P_{\text {drop }}$ ).

\section{THE DYNAMIC ADAPTIVE ARCHITECTURE: DYNAA}

Figure 1 shows the dynamic adaptive architecture DYNAA proposed. In this paper, the focus is on application and network adaptive layer.

\subsection{Application Layer}

The resource specification for a flow of a class $i$ specifies the minimum $b_{i, \min }$, the required $b_{i, \text { req }}$ and the maximum $b_{i, \max }$ granted bandwidth. Thus, the network has the ability to adjust the granted rate of the flow within the range of the resource specification. Multimedia applications must adopt the layered coding approach in such a way that they can accept varying degrees of 
network guarantee levels. When the adaptation handler informs the multimedia scaler about the granted bandwidth, the scaler selectively chooses a subset of the hierarchical coding depending on the resource availability. A customer in class $i$ uses one bandwidth among the range $\left[b_{i, 1}, b_{i, 2}, \ldots, b_{i, j}, \ldots, b_{i, K i}\right]$, where $b_{i, j}<b_{i, j+1}$ for $j=1,2 \ldots, K_{i}-1$. Note that $K_{i}$ is the number of class $i$ multimedia layers and $b_{i, j}$ the $j^{\text {th }}$ layer bandwidth of class $i$. According to the flow's resource specification, $b_{i, 1}$ is the minimum bandwidth $b_{i, \text { min }}, b_{i, j}$ the required bandwidth $b_{i, \text { req }}$ and $b_{i, K i}$ the maximum bandwidth $b_{i, \max }$ of class $i$.

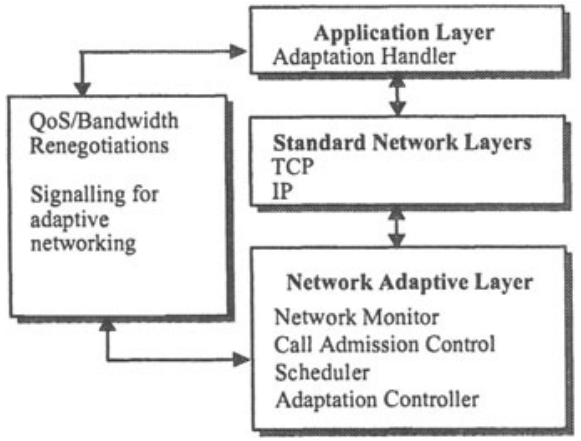

Figure 1. DYNAA Architecture

Applications define a softness profile that allows an efficient match of application requirements to network resource availability. According to the softness profile, we consider two classes of applications:

1. The Hard Adaptive class (HA): This class regroups applications that are adaptive with stringent constraints.

2. The Soft Adaptive class (SA): This class includes applications such as email and HTTP that are adaptive with soft constraints.

\subsection{Network Adaptive Layer}

The network adaptive layer implements specialized modules that support the multimedia requirements. These modules are the network monitor, the call admission control, the scheduler and the adaptation controller. In order to provide adaptive service, various algorithms interact in the following sequence of events (figure 2):

The network monitor computes periodically the amount, the ratio of application degradation and the current network load. These measures are used by the CAC when accepting new and HO calls. Application notifies the network that it wishes to set-up a flow between end-points and provides the flow specification. At this point, the network performs admission control using measures computed by the network monitor. After accepting a request, 
CAC communicates with the scheduler which schedules the waiting requests.

An incoming flow may cause a resource conflict between competing flows. Hence, the scheduler interacts with the adaptation controller in order to perform a resource adaptation among the existing flows. Next, the adaptation controller calls the bandwidth allocation algorithm $(B A A)$ or the bandwidth adaptation with no degradation algorithm (BNDA) for the distribution of bandwidth among competing flows.

DYNAA is a centralized/distributed architecture, using a centralized adaptation controller and distributed adaptation handlers. The adaptation controller notifies the adaptation handler about the bandwidth allocated through signalling. As for the adaptation handlers, they determine whether or not the application will adapt to any portion of the available bandwidth.

\subsection{CAC and Bandwidth Adaptation Algorithms}

The class $i$ degradation parameters $D D_{i}, D R_{i}$ and $P_{\text {drop }}$ measured periodically by the network monitor are passed to the CAC. We refer to $D D_{i, q o s}$ and $D R_{i, q o s}$ as the upper-bound values of the degradation parameters of a class $i$. Let $x_{i}(t)$ stands for the number of calls of class $i$ users in a cell at time $t . b_{i, a s s i}(s, t)$ denotes the assigned bandwidth for a call $s$ of class $i$ users at time $t$ where $b_{i, a s s i}(s, t) \in\left[b_{i, 1}, b_{i, 2}, \ldots, b_{i, j}, \ldots, b_{i, K i}\right]$ and $l \leq s \leq x_{i}(t)$. Let $I(f)$ be the indicator function which returns 1 if $f$ is true and 0 otherwise. If $n$ is the number of classes, $\Delta T$ the measurement time interval, and $\tau$ a time variable, the degradation parameters $D D_{i}$ and $D R_{i}$ of a class $i$ are such that [3]:

For $i=1, \ldots, n$ :

$$
\begin{aligned}
& D D_{i}(\tau)=\frac{1}{\Delta T} \int_{\tau-\Delta T}^{\tau} \frac{\sum_{k=1}^{x_{i}(t)}\left(b_{i, \text { req }}-b_{i, a s s i}(k, t)\right) I\left(b_{i, a s s i}(k, t)<b_{i} \text {,req }\right)}{\left(b_{i} \text {,req }-b_{i, 1}\right) \sum_{k=1}^{x i(t)} I\left(b_{i} \text { assi }(k, t)<b_{i} \text { req }\right)} d t \\
& D R_{i}(\tau)=\frac{1}{\Delta T} \int_{\tau-\Delta T}^{\tau} \frac{\sum_{k=1}^{x i(t)} I\left(b_{i}, a s s i(k, t)<b_{i}, \text { req }\right)}{x_{i}(t)} d t
\end{aligned}
$$

In our CAC, a new call is accepted only if the degradation parameters of all classes are less than the corresponding upper-bounds values. For HO calls, we introduce two thresholds for the call dropping probability: $P_{\text {drop_min }}$ and $P_{\text {drop_max }}$. If $P_{\text {drop }}$ is greater than $P_{\text {drop_max }}$ then the HO load is relatively high. Hence, the $\mathrm{HO}$ call is accepted without testing the degradation parameters and $B A A$ is applied. On the other hand, if $P_{\text {drop }}$ is less than $P_{\text {drop_min }}$ (respectively between $P_{\text {drop_min }}$ and $P_{\text {drop_max }}$ ), we accept the call if the performance degradation parameters are less than the upper-bound values and $B N D A$ (respectively $B A A$ ) is applied. The scheme proposed aims to have a compromise between $P_{d r o p}$ and the degradation performance parameters: 


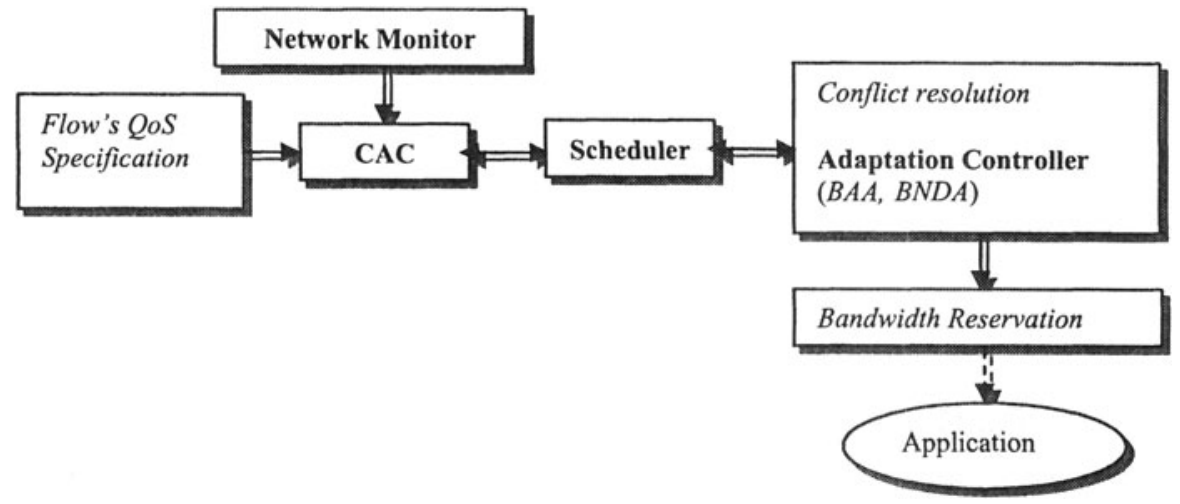

Figure 2. Network adaptive layer

In fact, the adaptation envisioned in this paper is adapted to the mobility through measuring $P_{\text {drop }}$.

$B A A$ and $B N D A$ will decide the changes for the calls' bandwidth in a cell adaptively when there is a call arrival. In order to prioritize HA class, the adaptation is performed over the SA class first and then over HA. In our paper, class 1 refers to $\mathrm{HA}$ and class 2 to $\mathrm{SA}$.

With $B A A$, if the available bandwidth $(A)$ is less than the required bandwidth $\left(b_{i, r e q}\right)$ of a new call belonging to the class $i$, our algorithm tries to lower the bandwidth of some calls belonging to SA class to $b_{2, \text { req. }}$ Then, the same procedure is applied to some calls of HA class. If $A$ is still less than $b_{i, r e q}$, the call is rejected whenever it is a new call. In case of a $\mathrm{HO}$ call, $B A A$ tries to squeeze to $b_{2, \min }$ the bandwidth of some calls belonging to SA class and then that of some calls of HA.

As for $B N D A$, some adaptation is made without call's degradation. In fact, $B N D A$ behaves as $B A A$ but differs in the $\mathrm{HO}$ handling. If after reducing to the required bandwidth, $A$ is still less than $b_{i, \text { reg, no degradation is }}$ performed. More details of the implemented algorithms are reported in [8].

\section{NUMERICAL RESULTS}

\subsection{Scenarios and Performance Evaluation}

The first scenario implements DYNAA as proposed in the previous section. In this scenario (referred as DYNAA), we don't assume that the HO requests can wait in queues. We have shown by simulation in [8] that DYNAA improves the overall performance. The second proposed scenario (referred as DYNAA_wait) implements DYNAA architecture with a waiting alternative. In this scenario, the HO requests belonging to SA and HA class (SA_HO and HA_HO requests) wait in two distinct queues. 

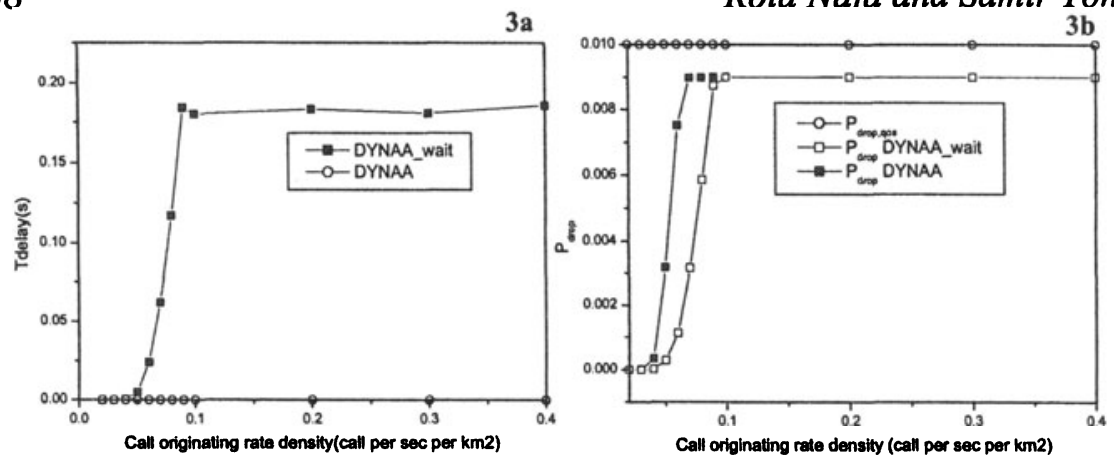

Figure 3. 3a. Mean average delay, 3b. Call dropping probability

A queued HA_HO request is deleted from the queue when it passes through the $\mathrm{HO}$ area before getting a channel or if its communication is completed before passing through the $\mathrm{HO}$ area. SA connections typically are more tolerant to delay as compared to HA connections. Thus, whenever a SA_HO request isn't satisfied within the current cell, it is transferred to the SA queue of the target cell.

Whenever there are some free resources due to a call departure, the new available bandwidth must be used in order to satisfy the $\mathrm{HO}$ requests and the degraded existing calls. Therefore, we apply the call admission control algorithm which tests $P_{\text {drop }}$ and the degradation parameters. Then, CAC communicates with the scheduler. In order to schedule the SA_HO and HA_HO requests, the Queue Length Threshold (QLT) scheduling policy is applied: QLT gives priority to SA traffic whenever the number of queued SA_HO requests is above some threshold $\left(L_{t h}\right)$ [9].

Afterwards, the scheduler interacts with the adaptation controller and applies $B A A$ or $B N D A$ according to the decision of the CAC. If after serving the $\mathrm{HO}$ requests, there are still some available resources, existing calls are upgraded as follows. First, we pick up the most degraded calls in the HA class and we increase their bandwidth to the required bandwidth. We keep doing this until there is no available bandwidth or until every call in the HA class has a bandwidth larger than or equal to $b_{l, \text { req }}$. Then, the same procedure is applied to SA class. Next, we try to increase the bandwidth of the calls with the smallest bandwidth in HA class. Same thing is done to SA class if the available bandwidth is still greater than zero.

Computer simulations have been derived by assuming a seven cell network. The edges of the simulated space wrap around to the opposite edges with each cell having a complete set of interfering cells so as to avoid the border effect. The considered cells are assumed to have a radius of $1 \mathrm{Km}$, and a capacity of 60 channels. Users are vehicular with an average speed of $40 \mathrm{Km} / \mathrm{h}$. The unencumbered session duration of a voice call is $120 \mathrm{~s}$. The bandwidth requirements are $\left(b_{1,1}, b_{1,2}, b_{1,3}\right)=(1,2,3)$ for class 1 (HA) and $\left(b_{2,1}, b_{2,2}, b_{2,3}\right)=(2,4,6)$ for class 2 (SA) such that $\left(b_{1, \text { req }}, b_{2, \text { req }}\right)=(2,4)$. 

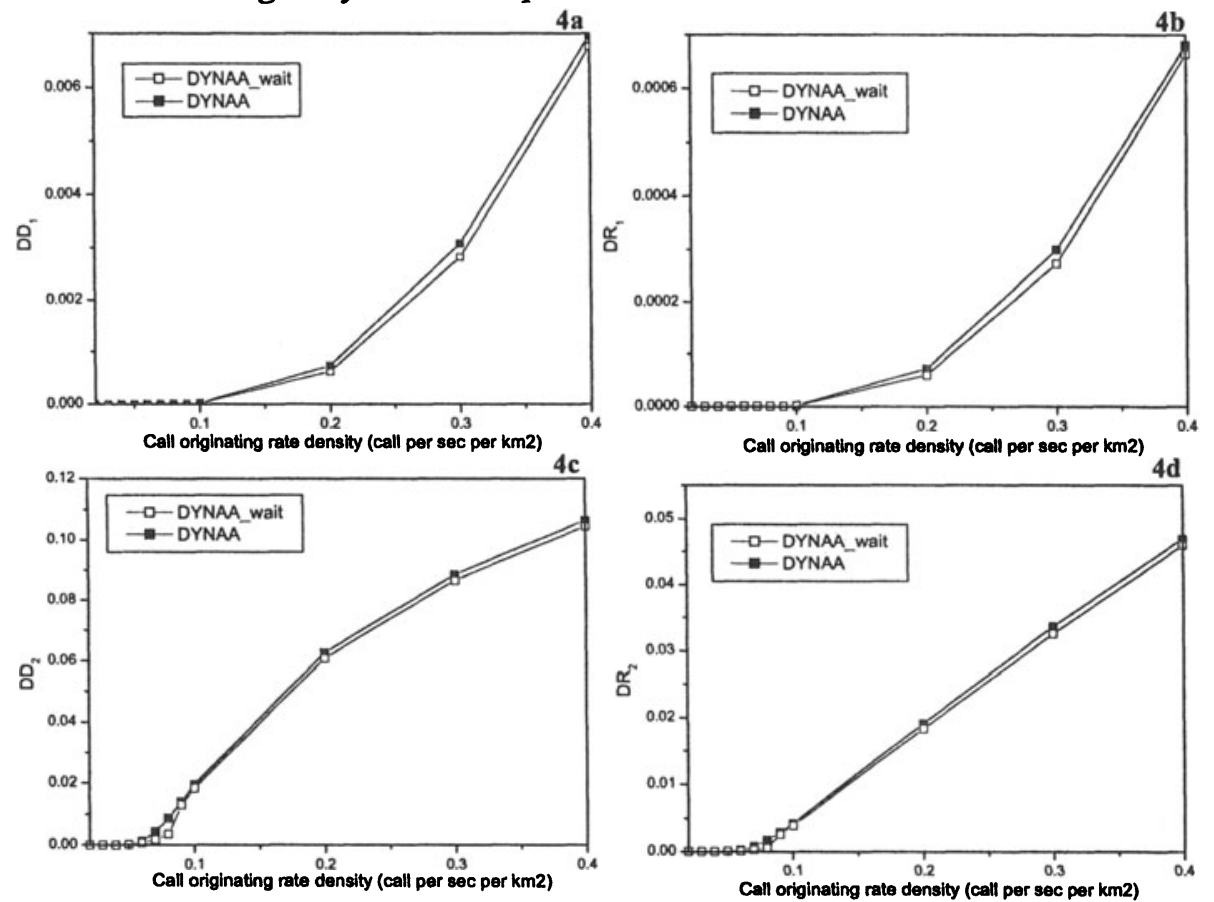

Figure 4. 4a,4b,4c,4d: Degradation Parameters of HA and SA classes

Note that the upper-bound values for $D D, D R$ are respectively $\left(D R_{1, \text { qos }}, D D_{1, \text { qoss }}\right)=(0.01,0.01)$ for $\mathrm{HA}$ and $\left(D R_{2, q o s}, D D_{2, q o s}\right)=(0.1,0.1)$ for SA. $\left(P_{\text {drop } \min ,}, P_{\text {drop } \max }\right)=(0.0075,0.009), P_{\text {drop, gos }}=0.01, L_{t h}=2$ and $\Delta T=5 s$.

The HA class in the simulation is represented by voice traffic. Its arrival process is assumed to be Poisson with mean rate $\lambda_{n v}$. The SA class considered in our paper is represented by a typical WWW session of type UDD $64 \mathrm{~Kb} / \mathrm{s}$ that consists of a sequence of packet calls [10]. The HTTP session arrival process is Poisson with mean rate $\lambda_{n d}$.

In this subsection, we try to compare the performance of DYNAA with DYNAA_wait scenario. The mean average waiting time $T_{\text {delay }}$ increases with DYNAA_wait (figure 3). This is quite logical because HO requests of the SA class wait before being served. On the other hand, with DYNAA_wait, more chance is given to the HO request to be served: $P_{\text {drop }}$ decreases due to the diminution of the forced termination of the calls. At the same time and as expected, DYNAA and DYNAA_wait manage to maintain $P_{\text {drop }}$ less than $P_{\text {drop_qos. }}$. At the call originating rate density of 0.1 calls per $\sec$ per $\mathrm{Km}^{2}, P_{\text {drop }}$ with DYNAA_wait becomes stable at the value of $0.9 \%$.

The priority of the HA class is reflected by its degradation values lower that those of the SA class (figure 4). The degradation parameters with DYNAA_wait are relatively less than those of DYNAA. In fact, since with DYNAA_wait the call dropping probability is reduced, the bandwidth with 
no degradation algorithm $(B N D A)$ is applied more frequently. On the other hand, the $\mathrm{HO}$ requests are served whenever there is a departure. Therefore, the HO requests will use the free resources to satisfy their need while HO requests in DYNAA do not have necessarily free resources to be served with. Thus, even if some priority is assigned to HO requests, degradation is relatively attenuated with DYNAA_wait scheme. Consequently, DYNAA_wait may improve the performance of DYNAA.

\section{CONCLUSIONS}

In this research, we investigated the adaptive resource allocation for multimedia applications. We proposed a dynamic adaptive architecture based on a CAC, a scheduler and bandwidth adaptation algorithms. It is shown that, by adjusting the amount of bandwidth degradation based on the current network conditions, our proposed scheme can be dynamic and consequently achieve better QoS. The proposed scenario has been extended by permitting to $\mathrm{HO}$ requests to wait in the $\mathrm{HO}$ area before being served. As a result, the overall performance has been improved.

\section{References}

[1] A.T.Campbell, R. R.-FLiao ,G. Bianchi, On Utility-Fair Adaptive Services in Wireless Networks, Sixth International Workshop on Quality of Service(IWQoS 98), 1998.

[2] T.Kwon, Y.Choi, C.Bisdikian, M.Naghshineh, Call Admission Control for Adaptive Multimedia in Wireless/Mobile Networks, Proceedings of ACM workshop on Wireless Mobile Multimedia,WOWMOM'99, Page(s): 51 -58.

[3] Y.Xiao, C.L.P. Chen, Y.Wang, Fair Bandwidth Allocation for Multi-class of Adaptive Multimedia Services in Wireless/mobile networks, IEEE 53rd Vehicular Technology Conference VTC 2001, Page(s): 2081 -2085 vol.3.

[4] S. Kim, T. Kwon,Y. Choi, Call Admission Control for Prioritized Adaptive Multimedia Services in Wireless/mobile Networks, IEEE $51^{\text {st }}$ Vehicular Technology Conference Proceedings VTC 2000, Spring Tokyo, Volume: 2 ,Page(s): 1536 -1540 vol.2.

[5] J.Wang, P.Cao, X.Yang, Adaptive Mobile Multimedia QoS Control and Resource Management, Ninth IEEE International Conference on Networks Proceedings, 2001.

[6] Y.Xiao, C.L.P Chen, QoS for Adaptive Multimedia In Wireless/Mobile Networks, Proceedings. Ninth International Symposium on Modeling, Analysis and Simulation of Computer and Telecommunication Systems 2001 Page(s): 81 -88.

[7] S.K.Das, S.K.Sen,P.Agrawal, K.Basu, Modelling QoS Degradation in Multimedia Wireless Networks, IEEE International Conference on Personal Wireless Communications, 1997 Page(s): 484 -488.

[8] R.Naja, S.Tohmé, DYNAA: DYNamic Adaptive Architecture for Quality of Service Provisioning in Wireless Mobile Multimedia Networks, submitted to IFIP WG6.7 Workshop and Eunice Summer School on Adaptable Networks and Teleservices.

[9] R.Naja, S.Tohmé, QoS Provisioning and Handover Issues in Mobile Wireless Multimedia Networks, IEEE Workshop on Applications and Services in Wireless Networks 2002, Paris.

[10] European Telecommunications Standards Institute ETSI-Universal Mobile Telecommunications Systems UMTS; Selection procedures for the choice of radio transmission technologies of the UMTS-TR101 112 V3.2.0-1998-04. 